\title{
Analogue Computer Studies of the Growth Characteristics of Escherichia coli Following Dihydrostreptomycin Treatment
}

\author{
By B. J. HAMMOND, MARGOT KOGUT AND J. W. LIGHTBOWN \\ National Institute for Medical Research, Mill Hill, London, N.W. 7
}

(Accepted for publication 2 March 1967)

\begin{abstract}
SUMMARY
Cultures of Escherichia coli B which had received pretreatment with dihydrostreptomycin were considered to consist of two subpopulations corresponding to the two fractions of culture samples that did and did not survive through plating and incubation to produce visible colonies. No initial assumptions were made about the viability of the subpopulations at the time that the samples were taken. A general-purpose analogue computer was then used both in mathematical analysis and mathematical synthesis to investigate the growth characteristics of the survivors and non-survivors as functions both of time and of pretreatment.
\end{abstract}

\section{INTRODUCTION}

Previous investigations into the effects of dihydrostreptomycin (DHS) upon cultures of Escherichia coli $\mathrm{B}$ have established a technique (Kogut, Lightbown \& Isaacson, $1965 a$ ) which allows intracellular effects to be studied in a situation uncomplicated by continuous uptake of antibiotic. This involves removal of antibiotic from the culture medium, by filtration and washing, after various periods of aerobic growth in the presence of DHS. By using this technique, growth rates at the time of removal of DHS have been shown to be decreased by an amount dependent upon the concentration of antibiotic and the duration of prior treatment. These decreased growth rates are maintained for periods from 2 to $4 \mathrm{hr}$ and constitute an initial phase of inhibited but logarithmic growth, which then gives way to a second phase, during which the growth rates recover to that observed for untreated control cultures. In a further paper (Kogut, Lightbown \& Isaacson, 1965b) viable counts determined during the various stages of inhibition and recovery were reported and showed that the viable count per unit population was also initially decreased by an amount dependent upon the preceding DHS treatment. Despite the numerical scatter encountered with viable count data, it was evident that the viable count per unit population remained relatively constant during the period of constant decreased growth rate, but subsequently increased during the period of growth rate recovery.

The association of constant viable count per unit population with the initial phase of constant growth rate implies that those bacteria which do and those which do not give rise to visible colonies may be dividing at similar rates. Direct microscopic observation of $E$. coli B after removal from DHS treated cultures adds support to this conjecture. The present paper reports closer investigation of the possibility that the growth rates of all the bacteria are initially equally affected by the form of DHS treatment described, and it examines the subsequent growth rate behaviour of that 
subpopulation which gives rise to visible colonies and that subpopulation which does not. A preliminary account of this work has been communicated (Hammond, Kogut \& Lightbown, 1966).

\section{METHODS \\ Biological methods}

The organism used, Escherichia coli strain B, its maintenance, preparation of inocula, growth media used for experiments (mineral medium of McQuillen \& Roberts, 1954, plus $0.2 \%(\mathrm{w} / \mathrm{v})$ trisodium citrate and $0.2 \%(\mathrm{w} / \mathrm{v})$ glucose) and growth conditions ( $32^{\circ}$ and aerobiosis obtained by adequate shaking) were as described by Kogut $e t$ al. $1965 a)$.

The growth of the bacterial population was measured as increase in extinction $(E)$ at $500 \mathrm{~m} \mu\left(E_{500}\right)$ in a Hilger 'Uvispeck' spectrophotometer with optical cells of $5 \mathrm{~mm}$. light path, and recorded as logarithms to the base two (Finney, Hazlewood \& Smith, 1955) which allowed direct determination of the specific growth rate in doublings $/ \mathrm{hr}(\mu)$, (Herbert, Elsworth \& Telling, 1956). It had been shown previously (Kogut et al. $1965 a$, table 1) that the relationships between $E_{500}$ values, total counts and dry weight determinations for samples taken at different times during growth in the presence or absence of dihydrostreptomycin are reasonably constant. Dihydrostreptomycin sulphate (Distillers Biochemicals Co. Ltd., Speke, Liverpool, 1; potency 800 i.u./mg.) was added to cultures in the early exponential stage of aerobic growth $\left(E_{500}=0 \cdot 1-0 \cdot 3\right)$. Removal of dihydrostreptomycin from treated cultures was achieved by rapid filtration on membrane filters (Millipore; diameter $47 \mathrm{~mm}$. A.P.D. $0.45 \mu$ ) followed by three washings (with half the original volume each time) and resuspension in antibioticfree medium pre-warmed to the growth temperature. Viable (colony) counts were made by diluting appropriate samples in sterile growth medium without glucose and plating on complete growth medium solidified with $1 \%(\mathrm{w} / \mathrm{v})$ agar. Pour-plates were made in triplicate from each of two different dilutions and incubated $33-35^{\circ}$ for $48-72 \mathrm{hr}$. The colony counts for each sample were determined from the dilution giving totals of between 150 and 400 colonies and expressed as visible colonies $/ \mathrm{ml}$. original sample. Those organisms in the original sample which do not give rise to visible colonies are usually described as 'non-viable', but since the possibility arises that they initially retain a finite growth rate, the less anomalous term 'non-survivor' has been adopted. This term, when applied to the appropriate fraction of a culture, is intended to imply that a sample will show upon incubation under the defined conditions the corresponding loss of viable units, but it does not directly imply that the fraction is nonviable at the time when the sample is taken. The conjugate term 'survivor' is also used, and replaces the designation 'viable' in reference to those bacteria which do produce visible colonies upon incubation.

For convenience of use with a computer the measured values of extinction and survivor counts were embodied as data in the following forms. The values of $E_{500}$ for each experiment were numerically normalized to provide a value of unity at the time of removal of dihydrostreptomycin, and this value then defined on a linear scale as unit 'population'. The resulting values, in population units, were then used as the first of two variables representing the experimental data. Also for each experiment the survivor counts were divided by the corresponding values of $E_{500}$ and this provided an index proportional to the number of survivors per unit population. The value of this 
index (number of colonies obtained from $1 \mathrm{ml}$. culture $/ E_{500}$ value) in the case of control cultures was evaluated from seventeen experiments covering all stages of exponential growth (i.e. $E_{500}$ from 0.15 to 0.95 ) and was found to be $1.9 \times 10^{9}$ per $E_{500}$ with a standard deviation of $7.6 \%$. The value of each index obtained from survivor counts for treated cultures, when divided by the above value for control cultures, was termed the 'survivor fraction'. Since it is impracticable to determine directly the standard deviations for each index obtained from treated cultures it had to be assumed that the standard deviation, expressed as a percentage, was the same in treated cultures and in controls. Error theory then gives the standard deviation for 'survivor fraction' as $11 \%$. Survivor fraction was taken as the second of the two variables embodying the experimental data.

\section{Computer methods}

The analogue computer installation that was used comprised a PACE TR 48 computer with both display oscilloscope and $X-Y$ recorder output facilities. These were used at $0 \cdot 1$ and 50 sec. compute times, respectively. Two different approaches, employing mathematical analysis and mathematical synthesis, were formulated on the computer; the methods are described in later sections where the various functions of time are described by the following symbols:

$$
\begin{aligned}
P & =\text { Total population (in population units) } \\
P_{s} & =\text { Survivor population (in population units) } \\
P_{n} & =\text { Non-survivor population (in population units) } \\
S & =\text { Survivor fraction (dimensionless) } \\
G & =\text { Growth rate of total population (doublings } / \mathrm{hr} \text { ) } \\
G_{s} & =\text { Growth rate of survivors (doublings/hr) } \\
G_{n} & =\text { Growth rate of non-survivors (doublings } / \mathrm{hr} \text { ) } \\
K_{s} & =\text { Rate constant at which survivor growth rate changes (per min.) } \\
K_{n} & =\text { Rate constant at which non-survivor growth rate changes, per min. }
\end{aligned}
$$

Method of mathematical analysis. Since the growth of a culture at any time may be described by

$$
d P / d t=0.693 G P,
$$

the evaluation of the growth rates of the survivors and non-survivors from the available data requires the numerical evaluation of

and

$$
G_{s}=\frac{1 \cdot 44}{P_{s}} \frac{d P_{s}}{d t} \quad \text { where } \quad P_{s}=P S
$$

$$
G_{n}=\frac{1 \cdot 44}{P-P_{s}} \frac{d\left(P-P_{s}\right)}{d t} .
$$

It is thus necessary to present to the computer for the analysis of each experiment the relevant data on total population and survivor fraction as measured at various times during the experiment. In the former case it was found sufficient to produce a voltage analogue of the data by setting a variable diode function generator (VDFG) to produce a straight-line approximation through the data curve at $40 \mathrm{~min}$. intervals. However the scatter of the data points of survivor fraction, which originates from the technique of viable counting, made it necessary first to draw a smooth curve through the survivor fraction data points and then approximate this latter curve by using a second VDFG. This method of analysis thus involves some pre-interpretation of the 
data. The required analysis also involves differentiation of functions derived from the VDFG outputs, and the straight-line approximations introduced by these devices, give rise to stepped derivative. The plotted solutions for the growth rates are correspondingly stepped and only the horizontal sections of the steps represent mathematically accurate solutions.

Method of mathematical synthesis. Since the preceding analytical approach involved a certain degree of subjective pre-treatment of the data the alternative approach using mathematical synthesis was also explored and a system devised which allowed a suitable range of hypotheses about the growth rates of two sub-populations, $P_{1}$ and $P_{2}$, to be implemented in the computer. For each set of hypotheses, $P_{1}$ and $P_{2}$ were provisionally considered as models of the subpopulations $P_{s}$ and $P_{n}$, respectively, and the validity of the assumption checked by comparing $P_{1}+P_{2}$ with total population data and $P_{1} /\left(P_{1}+P_{2}\right)$ with the data on survivor fraction. The general mathematical form adopted for the model populations was:

where

$$
d P_{1} / d t=0.693 G_{s} P_{1} \quad\left(t=0 ; \quad P_{1}=P_{s}(0)\right)
$$

and

$$
G_{s}=G_{s}(0)+\left[G_{s}(\infty)-G_{s}(0)\right]\left(1-\exp . K_{s}(T-t)\right),
$$

where

$$
d P_{2} / d t=0.693 G_{n} P_{2} \quad\left(t=0 ; \quad P_{2}=P_{n}(0)\right),
$$

$$
G_{n}=G_{n}(0)+\left[G_{n}(\infty)-G_{n}(0)\right]\left(1-\exp . K_{n}(T-t)\right) \quad\left(T<t ; \quad K_{s}=K_{n}=0\right)
$$

Examination of these equations as implemented in the patching diagram of Fig. 1 shows that apart from the initial sizes of the two populations, $P_{s}(0)$ and $P_{n}(0)$, all other parameters refer to the growth rates of the populations and each is represented on the computer by an independent potentiometer setting. Particular growth rate hypotheses may thus be readily implemented on the computer by appropriate settings of these potentiometers. For example, when constant growth rates are required the $T$ potentiometer may be set to correspond to a time interval greater than that of the duration of the experiment and the growth rate of $P_{1}$ will remain constant at any value set by the $\mathrm{G}_{s}(0)$ potentiometer. Correspondingly, $P_{2}$ will grow at any constant rate set by the $G_{n}(0)$ potentiometer. When the growth rates are required to vary from time zero, the $T$ potentiometer may be set to zero and the growth rate of $P_{1}$ will then change exponentially from the value set for $G_{s}(0)$ to any value set for $G_{s}(\infty)$ where the rate constant of the change is determined independently by the setting of the $K_{s}$ potentiometer. Similarly, the growth rate of $P_{2}$ will change from $G_{n}(0)$ to $G_{n}(\infty)$ at a rate constant of $K_{n}$.

The method used for checking the results of growth rate hypotheses against data was simply to pre-plot on paper the total population and survivor fraction data for any given experiment, and to superimpose over these, by using the computer $X-Y$ plotter, a trace of the corresponding curves computed for the particular set of growth rate hypotheses. In the case of pre-plotting the data on survivor fraction the limits set by its standard deviation were taken as the significant values. 


\section{RESULTS}

\section{Experimental data}

As reported in preceding papers (Kogut et al. 1965a,b;1966), addition of low concentrations of dihydrostreptomycin or streptomycin to aerobically growing cultures of Escherichia coli в during the early exponential phase led to intracellular accumulation of the antibiotic (also Hancock, 1962) and a gradual decline in growth rate as

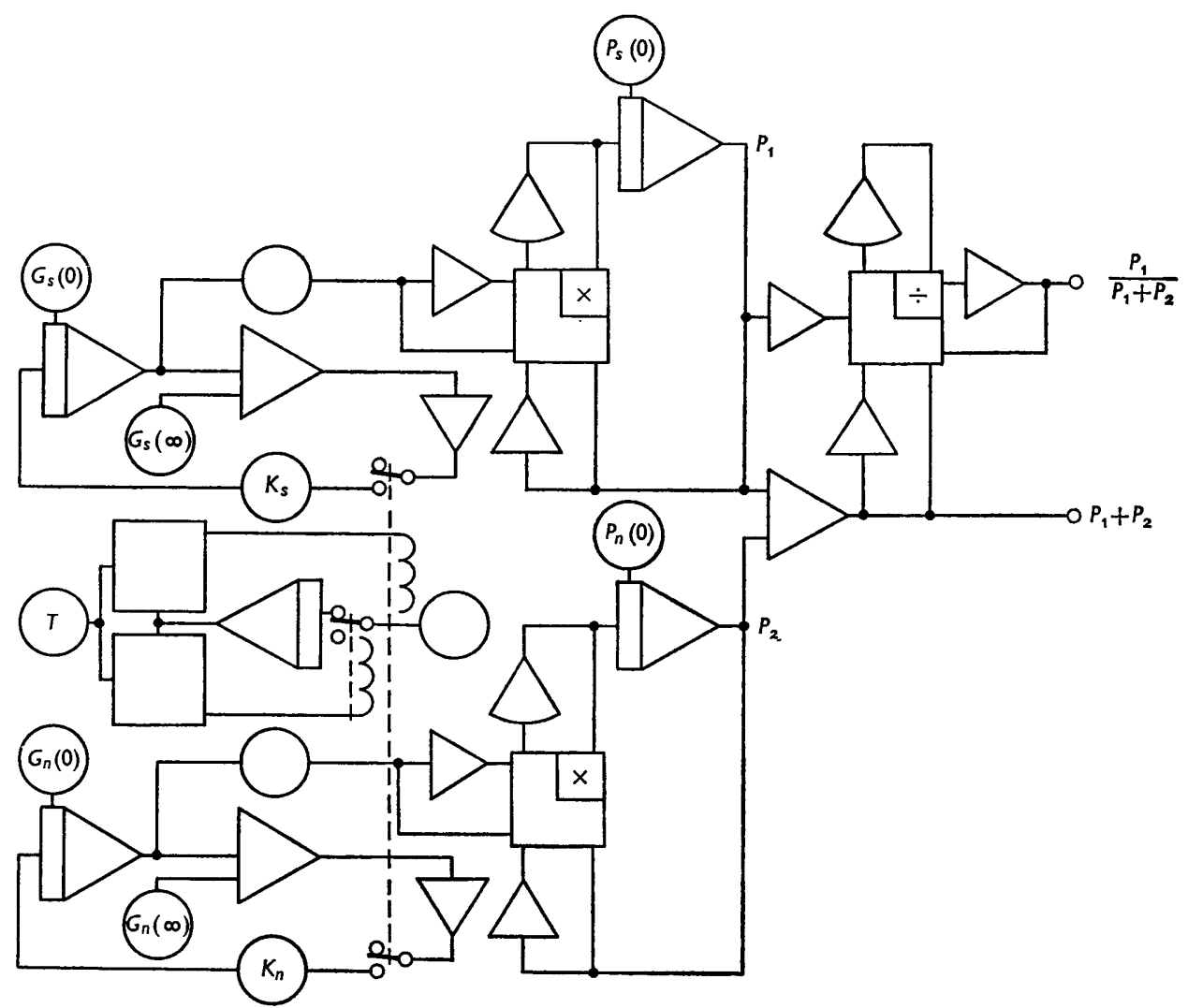

Fig. 1. Computer patching diagram. The above interconnexions of computer components generates curves for the theoretical populations $P_{1}$ and $P_{2}$ according to the equations given in the text. Outputs corresponding to $\left(P_{1}+P_{2}\right)$ and $P_{1} /\left(P_{1}+P_{2}\right)$ are also provided for comparison with experimental data. The constants of the equations appear as settings of the computer potentiometers as annotated.

illustrated in Fig. $2 a$ (see Kogut et al. 1965a). Removal of antibiotic from the culture medium at various times before growth had entirely ceased, prevented further intracellular accumulation and resulted in cultures which continued to grow at apparently exponential though decreased rates for several hours, followed by gradual recovery to normal growth rates; this is illustrated in Fig. $2 b$. We have already shown (Kogut et al. 1965b) that concomitant with the decrease in growth rate, the ratio of colonyforming units/ml. to $E_{500}$ values, i.e. the index of survivor count per $E_{500}$ value and hence the 'survivor fraction', as defined above, is decreased. After removal of extra- 
cellular antibiotic, the index of survivor count per $E_{500}$ was shown to remain more or less constant during the period when growth rate appeared constant, followed by gradual increase to the control value.

To obtain more complete data for mathematical analysis, we have now made measurements of survivor counts and $E_{500}$ values at frequent intervals to cover the whole of the initial logarithmic growth phase and the subsequent recovery phase of

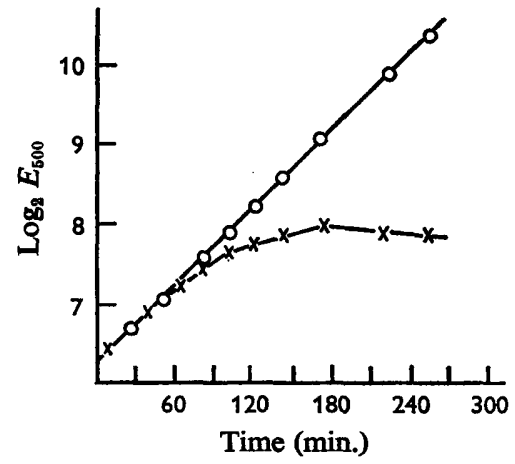

(a)

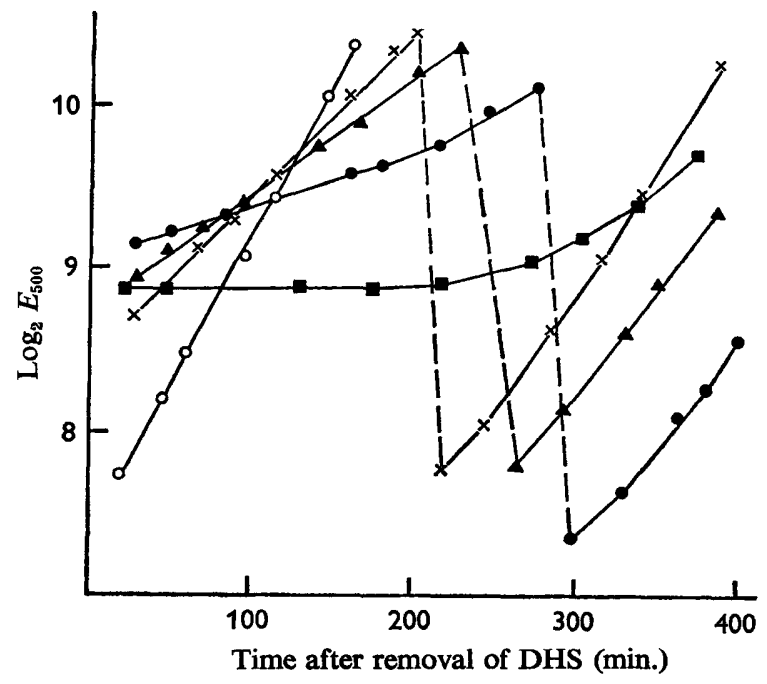

(b)

Fig. 2 (a) Growth curve of Escherichia coli B with and without the addition of dihydrostreptomycin sulphate, $20 \mu \mathrm{g} . / \mathrm{ml}$. at time zero. Growth conditions and measurements as described under Methods. $\mathrm{O}-\mathrm{O}$, control culture; $x-x$, culture with dihydrostreptomycin sulphate. (b) Aerobic growth of $E$. coli B in antibiotic-free medium after various periods of aerobic growth in the presence of dihydrostreptomycin sulphate, $20 \mu \mathrm{g} . / \mathrm{ml}$. Conditions as described in Methods. The dashed lines indicate culture dilution with fresh medium to allow for continued growth. $O-O$ represents a control culture and the remainder, treated cultures subjected to various periods of pre-treatment and filtered after: $\times-\times, 65 \mathrm{~min} . ; \Delta-\Delta, 85 \mathrm{~min}$.; $-0,105 \mathrm{~min} ; 0-\square, 125 \mathrm{~min}$.

Table 1. Initial growth rates, survivor counts and survivor fractions of Eschericha coli $B$ cultures following treatment with dihydrostreptomycin (DHS) of various concentrations for various times

Expt. no....

Concentration of DHS

Duration of DHS treatment (min.)

Initial constant growth rate $(\mu)$

Decrease from control (\%)

Initial survivor count $/ E_{500}\left(\times 10^{\circ}\right)$

Survivor fraction
1 30

70

65

105

3

4

5

6

Control

Before filtration

$\begin{array}{rrrrrr}30 & 20 & 30 & 20 & 30 & 0 \\ 65 & 105 & 75 & 110 & 65 & 0\end{array}$

After filtration

$0 \cdot 17$

$0 \cdot 32$

0.35

$0 \cdot 37$

0.45

0.56

$1 \cdot 0$

83

68

65

63

55

44

1.4

0.37
0.20

0.76

0.96

$1 \cdot 1$

$1 \cdot 3$

0.74 
increasing growth rates and survivor fractions, on cultures treated with dihydrostreptomycin for various times and then freed from extracellular antibiotic. Figure 3 shows the time course for a representative experiment, and the relevant details for the six sets of experiments are tabulated in Table 1.

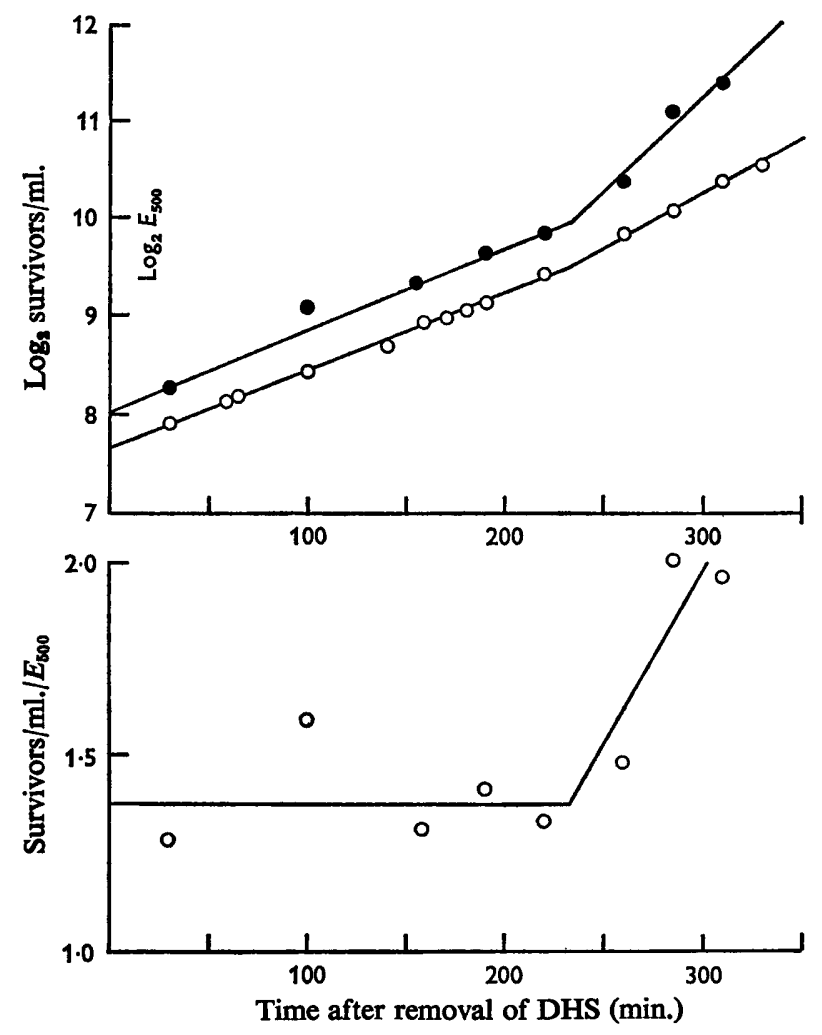

Fig. 3. The upper graph shows the increase in total population, measured as $E_{500}$, and the corresponding number of survivors $/ \mathrm{ml}$. in a culture of Escherichia coli B after treatment with dihydrostreptomycin sulphate, $20 \mu \mathrm{g} . / \mathrm{ml}$. for $110 \mathrm{~min}$. followed by removal of extracellular antibiotic (Expt. 5 of Table 1). $\bigcirc-0, \log _{2} E_{500} ; 0-0, \log _{2}$ survivors $/ \mathrm{ml}$. The lower graph shows the ratio between these variables, namely the number of survivors $/ \mathrm{ml}$./ $E_{500}$.

\section{Results of mathematical analysis}

The method of mathematical analysis previously described was applied to the six sets of experimental data and the corresponding stepped solutions for growth rates as functions of time obtained. A typical result is that of Fig. 4, where the derived values of growth rates of survivors and non-survivors are shown as points taken from the horizontal sections of the stepped solution curve. The numerical values of such points depend upon the particular smooth curve chosen to represent survivor fraction data in the computer and the results are taken to be qualitative rather than quantitative. The majority of solution curves obtained were of the same basic structure as Fig. 4 and it was noted that in each experiment the growth rate of the non-survivor population eventually assumed a negative and not merely a zero growth rate thus indicating a non-survivor population which decreased in size at later stages of the experiments. 


\section{Results of mathematical synthesis: 1; growth-rate parameters as functions of time}

The method of mathematical synthesis was initially used to investigate certain simple hypotheses involving constant growth rates. The first of these hypotheses was the case where the non-survivors are assumed to be completely non-viable, that is, their growth rate is zero, whilst the survivors have a constant growth rate throughout the experiment. This assumption covers the possibility that the survivors continued to grow at control growth rates. The hypothesis does not specify the initial sizes of the survivor and non-survivor populations, but these are substantially established by the initial

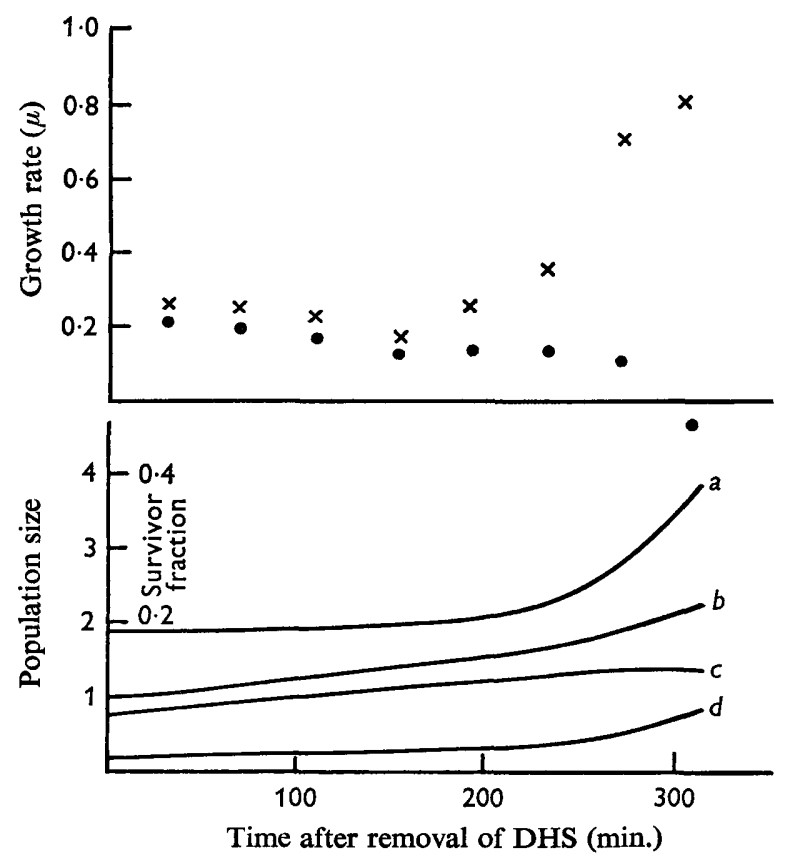

Fig. 4. Curve $a$ is the computer representation of the survivor fraction data from Expt. 1 of Table 1, and is obtained from a suitably preset VDFG unit: curve $b$ is the representation of the corresponding total population data similarly obtained. These data curves, analysed according to the equations given in 'Methods of mathematical analysis', provide the following solutions: curve $c$, size of non-survivor population; curve $d$, size of survivor population; - - , growth rate of non-survivor population; $x-\times$, growth rate of survivor population. The latter growth-rate solutions are shown only as the mathematically accurate points of the computed curve in which steps occur due to mathematical artifact.

values of total population and survivor fraction data. The only variable is thus survivor growth rate and this was varied on the computer until the hypothesis produced a match to total population data. The situation is illustrated in Fig. $5 a$, which relates to Expt. 1 where a DHS concentration of $30 \mu \mathrm{g} . / \mathrm{ml}$. had been administered $70 \mathrm{~min}$. before time zero. A value of survivor growth rate of 0.540 doublings $/ \mathrm{hr}$ was found necessary to produce a match to total population data, and the corresponding computed survivor fraction curve was as shown. Any attempt to bring the latter into closer agreement with its data involved an unacceptable divergence of the total population curve. 
The second of the hypotheses involving constant growth rates removed the restriction that the value of the constant for the non-survivor population should be zero, and thus presumed a total population that was the sum of two independent logarithmic subpopulations. Neither of the growth rates is specified in the hypothesis, but these were adjusted on the computer to determine the combination which gave a total
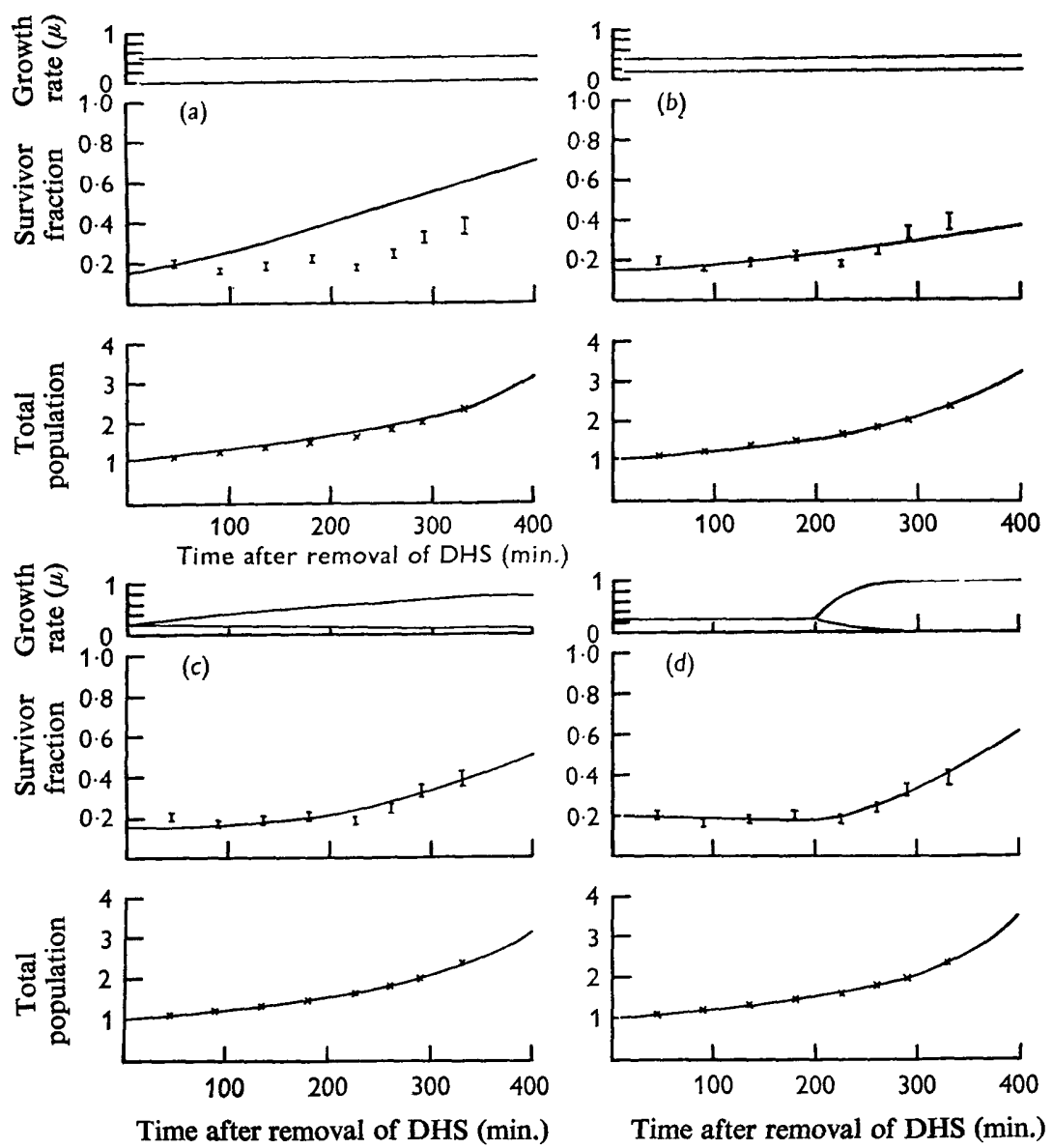

Fig. 5. Four comparisons between the curves synthesized from various growth-rate hypotheses and the experimental data of Expt. 1, Table 1. In all cases the time axes refer to time after removal of extracellular antibiotic, and in each case the growth-rate hypotheses are embodied in the upper curves of each section. The centre graphs of the four sections show the experimental data points for survivor fraction (I) and the solid curves indicate the computed result of growth-rate hypotheses. The corresponding computed curves for total population are shown in the lower graphs of each section together with the total population experimental data $(x)$.

population curve that fitted the data. Figure $5 b$ shows the resultant curves for the same experiment as previously considered, where the determined values of growth rates were 0.400 and 0.160 doublings/hr for survivors and non-survivors respectively.

The remaining hypotheses that were tested all involved varying growth rates and these were implemented on the computer as exponential changes from some initial 
value towards a second and final value. The final value for the survivor growth rate was made equal to the approximate control value of 1 doubling $/ \mathrm{hr}$, thus allowing for the observed eventual recovery of a treated population; that for the non-survivors was made zero, thus allowing for their eventual non-viability. Since the rates of the growth rate changes could be varied by potentiometer control on the computer and the adjustment included the possibility of setting the rates to zero, this system allowed the possibility that the non-survivor growth rate was zero.

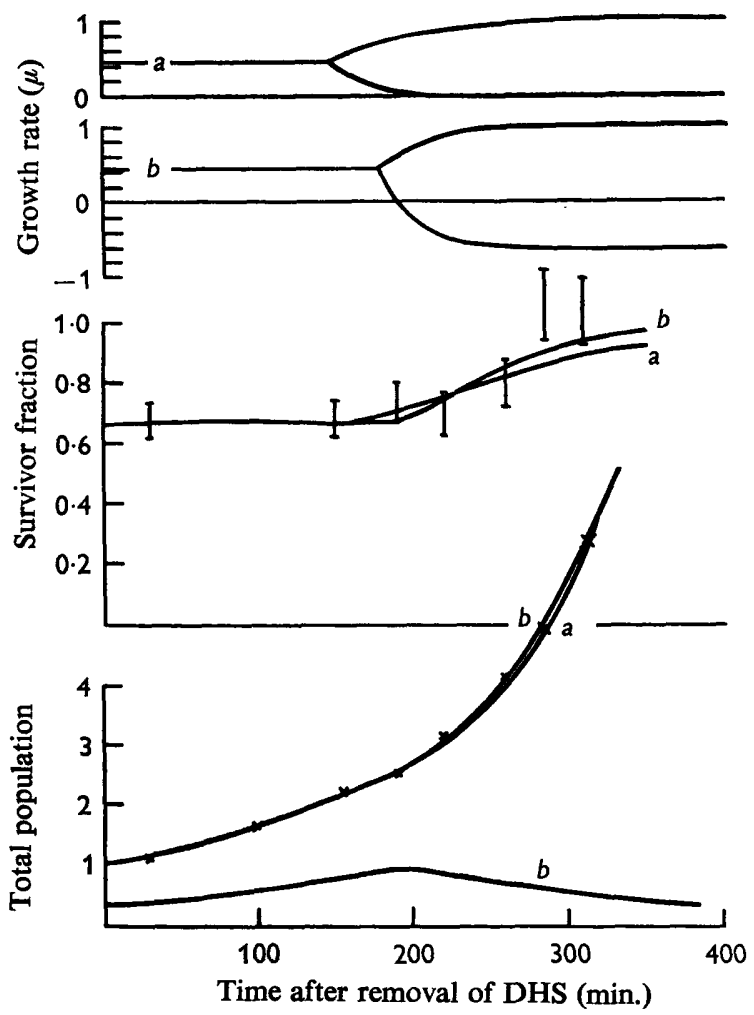

Fig. 6. Comparison of growth-rate hypotheses with the data of Expt. 5, Table 1. The upper graphs, $a$ and $b$, represent variant hypotheses regarding the ultimate non-survivor growth rate and the centre graph compares the survivor fraction data (I) with the computed implications of these hypotheses. The lower graph makes simultaneous comparison between the total population data $(x)$ and the computed curves. The bottom curve records the nonsurvivor population corresponding to the second hypothesis $(b)$.

Figure $5 c$ shows the situation that resulted from a hypothesis which allowed the growth rates of survivors and non-survivors to start their exponential changes as soon as DHS was removed from the culture medium. The experiment illustrated is the same as that previously used and the best match to its data was achieved by adjusting the value of initial growth rate and the subsequent rate constants of the changing survivor and non-survivor growth rates. The value of the initial growth rate was found to be 0.20 doublings $/ \mathrm{hr}$ and those of the rate constants were $0 \cdot 18(4) / \mathrm{min}$. and $0 \cdot 11(2) / \mathrm{min}$., respectively. These gave rise to the recorded growth rate and survivor fraction curves.

The final type of hypothesis considered differed only from the above in that an un- 
specified period was allowed before the onset of growth rate change, during which the survivor and non-survivor growth rates were constant and equal. The period of this delay, together with the initial value of growth rate and the rate constants of ensuing growth rate changes were treated as parametric variables in finding the set of values which not only fitted the total population data but also gave the best fit to survivor fraction data. The values so determined in the case of Expt. 1 were $200 \mathrm{~min}$. for the delay time, an initial growth rate of 0.20 doublings $/ \mathrm{hr}$ and $2 \cdot 2(2) / \mathrm{min}$. and $2 \cdot 0(9) / \mathrm{min}$.
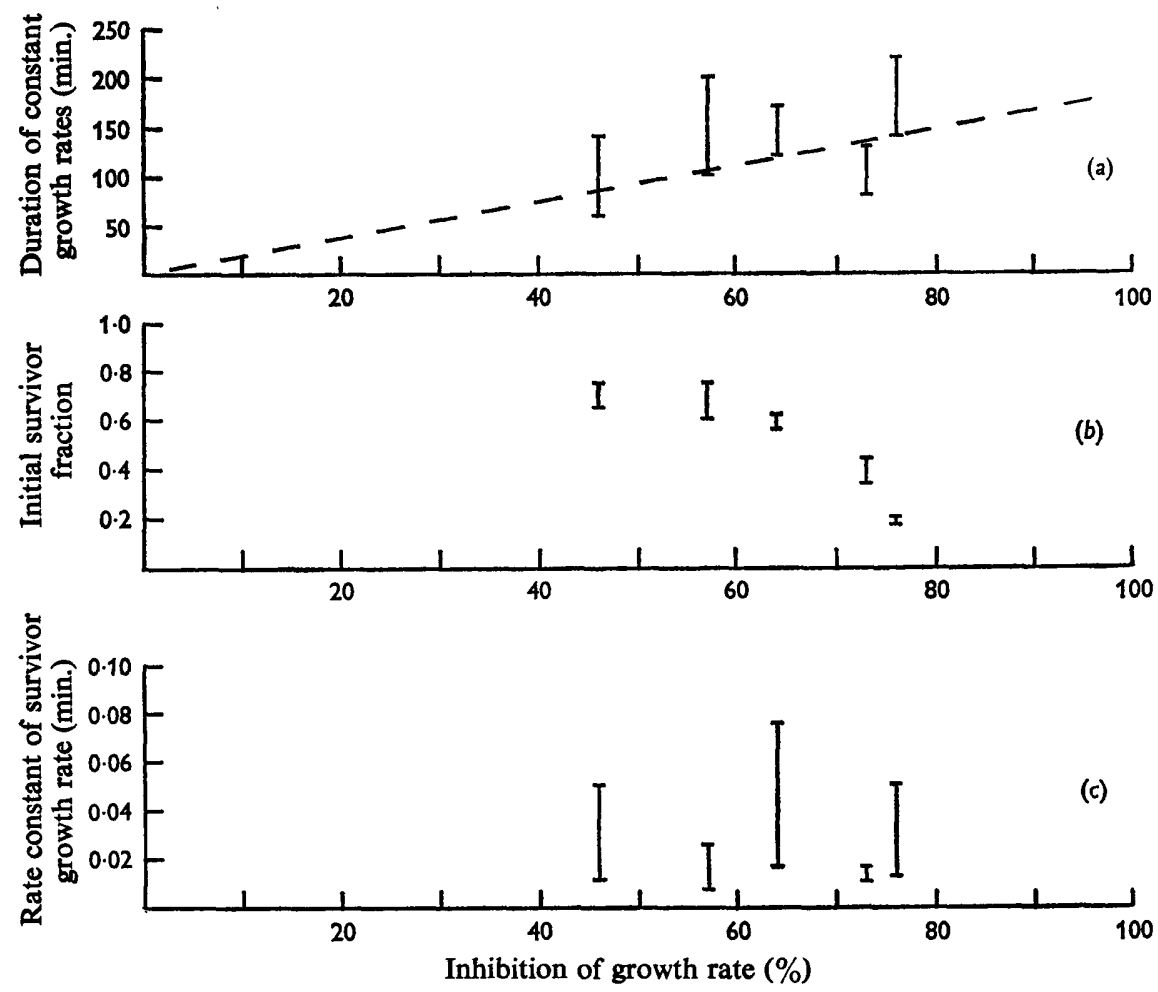

Fig. 7. The maximum and minimum values of the indicated parameters were determined as described in 'Results of mathematical synthesis, 2' and the values so determined are represented by bars at the ends of the vertical lines. These values are presented as functions of the growth-rate inhibition that results from various concentrations and times of dihydrostreptomycin pretreatment. A maximum value of one parameter is associated with maxima of the other two parameters. Results from only five of the six experiments are shown since those from experiments three and four were almost identical.

for the rate constants of the ensuing survivor and non-survivor growth rates changes, respectively. The forms of the resultant growth rate curves, total population and survivor fraction curves are shown in Fig. $5 d$.

Similar results to these were obtained for all six sets of data with, however, a tendency for less satisfactory final fits to be obtained for those experiments involving higher initial survivor fractions. Such an experiment is no. 5 of Table 1; the situation is shown in Fig. 6 which illustrates the improvement in fit that resulted when the eventual growth rate of the non-survivors was allowed to become negative and not merely zero. 
Results of mathematical synthesis: 2; growth rate parameters as function of treatment

Each set of data was re-examined by using the computer simulation of the hypothesis involving a 'delay' period of equal and constant survivor and non-survivor growth rates before the onset of exponential changes. In this study however, the maximum and minimum values for the delay period, which by suitable adjustment of other parameters would still allow acceptable fits to both total population and survivor fraction data, were examined. It was found that to obtain a maximum delay period which still allowed such acceptable fits to experimental data, it was necessary to use on the computer a maximum value of initial survivor fraction and a maximum value of the rate constant of survivor growth rate recovery. The converse situation applied when a minimum initial period of constant growth rate was enforced. Other parameters were inconsistently related to the maximum and minimum delay situations. It was thus possible to determine for each experiment maximum and minimum values for initial survivor fraction, duration of period of constant growth rates, and the rate constant of survivor growth rate. These values are plotted as functions of the initial value of constant growth rate in Fig. $7 a-c$ : only five sets of values are given since those from Expts. 3 and 4 of Table 1 were found to be almost identical.

\section{DISCUSSION AND CONCLUSIONS}

\section{Growth rate parameters as functions of time}

The original suggestion (Kogut et al. 1965 b) that the early effects of dihydrostreptomycin upon growth rates of Escherichia coli B cultures were similar in all individuals was based upon consideration of growth characteristics and viable counts of cultures freed from extracellular antibiotics, and was supported by direct microscopic observation. This proposition, which contrasts with the more common assumption that loss of viability consequent upon antibiotic treatment implies immediate and complete cessation of growth (Hurwitz, Landau \& Doppel, 1962) was made despite the ensuing separation of a bacterial population into two fractions, according to the retention or loss of the capability to form a colony when incubated for $72 \mathrm{hr}$.

The apparent anomaly that bacteria whose growth rates were initially equally affected, nevertheless ended as members of one or other of these two opposed categories ('viable' and 'non-viable') was recognized at the time and resolution of the anomaly sought in attributing genuine changes of growth rate to the two groups. It was also observed that the loss of ability to go on dividing did not occur throughout the $72 \mathrm{hr}$ period of incubation, but seemed to become established within the first 3-4 generation times after removal of extracellular dihydrostreptomycin.

The nature of the changes in growth rates, postulated for the two subpopulations, may in principle be determined analytically, by using the simple equations previously described; but errors inherent in one of the independent variables of the equations, namely the survivor fraction data, eventually limits use of the method to qualitative interpretation. It should not be overlooked, however, that the solutions obtained do approximate to the unique solution for each experiment, and such results as those in Fig. 4 do strongly support the original contentions about the form of growth rate variations for survivors and non-survivors.

Further indirect support for the proposed growth characteristics is obtained by 
means of mathematical synthesis, which shows that some simpler conjectures involving constant growth rates are untenable, on the basis that their quantitative results cannot be made to match experimental data (Figs. 5a,b). Good agreement with data, however, can be obtained (Fig. $5 d$ ) with the mathematical formulation of the hypothesis that the growth rates of all individuals in the culture have an initial depressed value which remains constant for a period, followed by a second phase when the growth rate of the survivors increases exponentially to the control value, and that of the non-survivors decreases exponentially to zero. No intrinsic significance is attributed to the exponential form of growth-rate change as this form was merely chosen for convenience; but the overall sigmoid form of growth rate changes is proposed as being a close representation of the true situation.

Both the methods used, mathematical analysis and mathematical synthesis, give indication that the growth rates of non-survivors may eventually assume negative values, implying that the number of non-survivors in such cultures actually decreases towards the end of the experimental period. This tendency is most apparent in those cultures where the initial common growth rate and the size of the initial survivor fraction were least severely depressed. The simplest explanation of such decrease in the number of non-survivors would lie in the eventual lysis of some, if not all, of the non-surviving bacteria. Lysis of even substantial fractions $(20-40 \%)$ of the total population, occurring during the period when growth rates of total population and of the survivor fraction were increasing would be impossible to detect by extinction measurements. (We only occasionally observed decrease in $E_{500}$ values of cultures that had stopped growing and were left in the presence of extracellular dihydrostreptomycin.) In four determinations of $E_{500}$ values and total counts on cultures which were left in the presence of extracellular antibiotic after growth had completely ceased, the ratio between these two increased, i.e. the bacteria appeared to shrink or become denser. Alternative explanations for the possible decrease in number of non-survivors can be derived on the basis of differences between growth characteristics of Escherichia coli in liquid culture and on solid media. It could, for instance, be assumed that a loss or excretion of antibiotics from some of the bacteria during growth in liquid culture, where extracellular dilution would be rapid might lead to recovery of the ability to go on dividing, whilst such recovery would be prevented by the slower diffusion of such excreted antibiotic in solid media. In such a situation successive samples from a liquid culture would score decreasing numbers of non-survivors upon incubation on solid media. Moreover, such a phenomenon would be most apparent in cultures which show less severe depression of initial growth rate and survivor fraction, and presumably have smaller total intracellular concentrations of antibiotic (Kogut, Lightbown \& Isaacson, 1966).

\section{Growth parameters as functions of treatment}

A consistent relationship between pre-treatment with dihydrostreptomycin, resultant survivor fraction and the initial growth rate of the corresponding total population was previously shown (Kogut et al. 1965b). The duration of common constant growth rates in cultures with various degrees of initial growth-rate inhibition cannot be determined with accuracy, but the results obtained here (Fig. 7a) are consistent with a linear increase from zero for an untreated culture at a mean rate of 15-20 min. $10 \%$ increase in growth-rate inhibition. Such a linear relationship would 
imply that the duration of common constant growth rate is not dependent on bacterial multiplication or on the amount of growth, but is consequent upon the same or related factors(s) which govern(s) the initial inhibition of growth rate.

The rate constants of survivor growth-rate recovery in some cases display very wide limits. The results in Fig. 7c, which gives these values as functions of initial growth rate inhibition, allow no specific relationship between these variables to be postulated.

The relationship between initial growth-rate inhibition and size of the survivor fraction, initially postulated, is not supported by the results shown in Fig. $7 b$. Any curve drawn through these points must also conform to the conditions of a control culture and have a unity value of survivor fraction at zero inhibition; the relationship between these two variables then becomes evidently non-linear. Extrapolation of any simple curve through the points also implies that pre-treatment with higher levels of dihydrostreptomycin for longer periods will produce bacterial populations consisting entirely of non-survivors, which can nevertheless retain an apparent initial growth rate (rate of increase in $E_{500}$ value) of approximately $20 \%$ of the control value. It must be remembered, of course, that this refers to the initial period of common constant growth rate which persists for about $3 \mathrm{hr}$, so that the corresponding increase in bacterial mass for that period would be about 50-60\%. Occasional direct determinations of protein (Lowry, Rosebrough, Farr \& Randall, 1951): $E_{600}$ ratios of cultures whose growth rate was severely inhibited (by 80-90\%) showed no significant decrease from control values. This makes it unlikely that such 'residual' growth rates merely reflect synthesis of carbohydrates or reserve materials.

It remains to consider mechanisms which might account for the growth patterns of populations of Escherichia coli B treated with streptomycin or dihydrostreptomycin in the manner described. If such treated populations were already differentiated in their growth behaviour at the time of removal of extracellular antibiotic, then one portion (the survivor fraction) should have growth rates which continuously increase and the other (the non-survivor fraction) should exhibit continuously decreasing growth rates. This situation is contradicted by the results of analogue computer simulations presented here. What then is the mechanism which results in approximately equal and constant growth rates which are maintained for periods extending through 3 to 4 generations? The present study confirms the previous suggestions that 'loss of viability' does not occur as a sudden and irreversible cessation of growth and multiplication, i.e. a unique 'lethal' event, at the time when the antibiotic enters the organism, but seems to be consequent upon a series of events taking place in the presence of intracellular antiobiotic. It has been suggested, on the basis of earlier studies, (Kogut et al. $1965 b ; 1966)$ that some of the intracellular antiobiotic accumulated by treated populations is not immediately inhibitory, but constitutes a 'pool' from which transfer and combination with the sites of inhibition can take place during subsequent growth in the absence of further intracellular accumulation. The distribution of such pools within a treated population need not be uniform. Elucidation of the factor(s) which may govern the transfer of intracellular antibiotic between 'pool' and the sites of inhibition (e.g. the ribosomes) would involve a better understanding of the nature and distribution of such non-inhibitory 'pool' streptomycin within the bacteria, as well as the nature of the effective growth-inhibitory combination between streptomycin and ribosomes and the control of ribosome synthesis and assembly. 
Our thanks are due to Miss Maureen Harris for able and conscientious technical assistance.

\section{REFERENCES}

Finney, D. J., Hazlewood, T. \& SMrth, M. J. (1955). Logarithms to base two. J. gen. Microbiol. $12,222$.

Hammond, B., Kogut, M. \& LightBown, J. W. (1966). Analogue computer studies of the growth characteristics of survivors and non-survivors in dihydrostreptomycin-treated cultures of Escherichia coli: the concept of lethality. J. gen. Microbiol. 44, vii.

HANCOCK, R. (1962). Uptake of ${ }^{14} \mathrm{C}$-streptomycin by some micro-organisms and its relation to their streptomycin sensitivity. J. gen. Microbiol. 28, 493.

Herbert, D., Elsworth, R. \& Telling, R. C. (1956). The continuous culture of bacteria; a theoretical and experimental study. J. gen. Microbiol. 14, 601 .

HuRwitz, C., LANDAu, J. V. \& DopPel, H. W. (1962). Effect of exposure of Escherichia coli to streptomycin on ability to undergo cell division. J. Bact. 84, 1116.

KogUt, M., LIGHTBOWN, J. W. \& ISAACSON, P. (1965a). Streptomycin action and anaerobiosis. J. gen. Microbiol. 39, 155.

KogUt, M., LiGHTBOWN, J. W. \& IsAacson, P. (1965b). Effects of dihydrostreptomycin treatment on the growth of Escherichia coli after removal of extracellular antibiotic. J. gen. Microbiol. 39, 165 .

Kogut, M., LightBown, J. W. \& IsaAcson, P. (1966). The intracellular accumulation of ${ }^{14} \mathrm{C}$-streptomycin by Escherichia coli strain B in relation to its growth-inhibitory effect. J. gen. Microbiol. 42, 333.

Lowry, O. H., Rosebrough, N. J., FarR, A. L., \& Randall, R. J. (1951). Protein measurement with Folin phenol reagent. J. biol. Chem. 193, 265.

MCQUILLEN, K. \& RoBERTs, R. B. (1954). The utilization of acetate for synthesis in Escherichia coli. J. biol. Chem. 207, 81. 